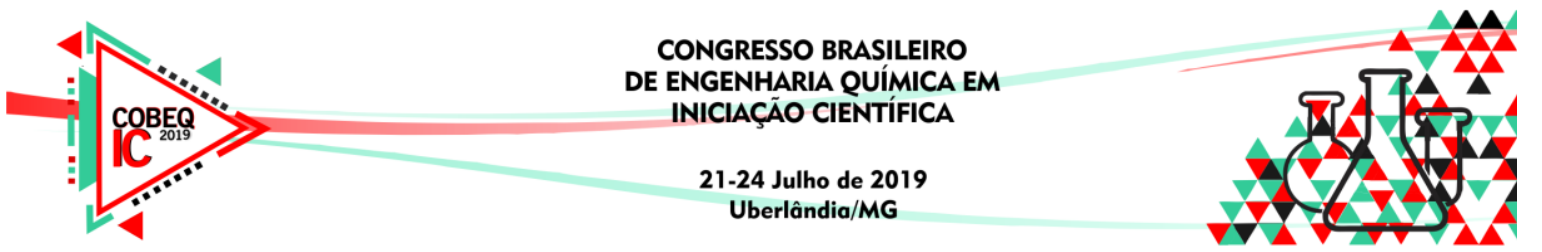

\title{
ESTUDO DA CINÉTICA DE ADSORÇÃO DO CORANTE EOSINA Y EM CARVÃO ATIVADO DE CASCA DE ARROZ
}

\author{
D. C. P. CRUZ ${ }^{1}$, R. L. C. SEHWARTZ ${ }^{1}$, R. M. LIMA ${ }^{1}$, R.L. PINA ${ }^{1}$, D. R. PINHEIRO ${ }^{1}$ \\ ${ }^{1}$ Universidade Federal do Pará, Instituto de Tecnologia, Faculdade de Engenharia Química \\ E-mail para contato: delispalhetac@gmail.com
}

\begin{abstract}
RESUMO - Neste trabalho se avaliou a adsorção de eosina Y em carvão ativado, obtido a partir da casca de arroz. O carvão ativado preparado em meio ácido apresentou ponto de carga zero (PCZ) igual a 3,28. Ensaios de cinética de adsorção utilizando $0,3 \mathrm{~g}$ de carvão e concentração de $200 \mathrm{ppm}$ de eosina foram realizados em meios ácidos e básico: $\mathrm{pH}=4$ e 8 , respectivamente. Os dados cinéticos foram correlacionados com modelos cinéticos de primeira e segunda ordem, cujos parâmetros foram obtidos via regressão não linear e com o auxílio do programa Origin. O critério de análise foi com base nos valores de $\mathrm{R}^{2}$. Os resultados indicaram que o método de preparo do carvão tem grande influência sobre o PCZ e consequentemente sobre a capacidade adsortiva.
\end{abstract}

\section{INTRODUÇÃO}

Com o crescente aumento do setor industrial têxtil, ocorreu a elevação da utilização dos corantes utilizados para o beneficiamento dos produtos. A Eosina-Y é um dos corantes mais conhecidos no setor têxtil, todavia, suas moléculas afetam severamente os seres humanos, podendo provocar danos nos rins, fígado e pulmões e aos olhos (Anitha et al 2016).

Dentre os processos empregados para a remoção dos contaminantes destaca-se a adsorção. A adsorção é uma operação de transferência de massa que estuda a capacidade de certos sólidos em concentrar na sua superfície determinadas substâncias presentes em fluidos, possibilitando a separação (Ruthven, 1984).

Atualmente o carvão ativado é uma das principais matérias-primas utilizadas no processo de adsorção devido sua elevada superfície especifica oriunda da característica micro, meso e macroporosa, obtida através da calcinação, o que propicia a retirada efetiva do adsorvato, gerando grande eficiência e baixa produção de rejeitos. Para viabilizar o processo pesquisadores buscam insumos de baixo custo; uma matéria prima de grande viabilidade é a casca de arroz, tendo em vista que conforme Reis (2015) no Brasil são produzidas 2,74 milhões de toneladas de casca de arroz anualmente, sendo utilizadas em sua maioria como material para geração de energia.

Neste contexto, o presente trabalho teve como objetivo sintetizar um carvão ativado a partir da casca de arroz, visando a remoção do corante Eosina Y de líquidos, além de identificar os modelos cinéticos que descrevem o processo de adsorção. 


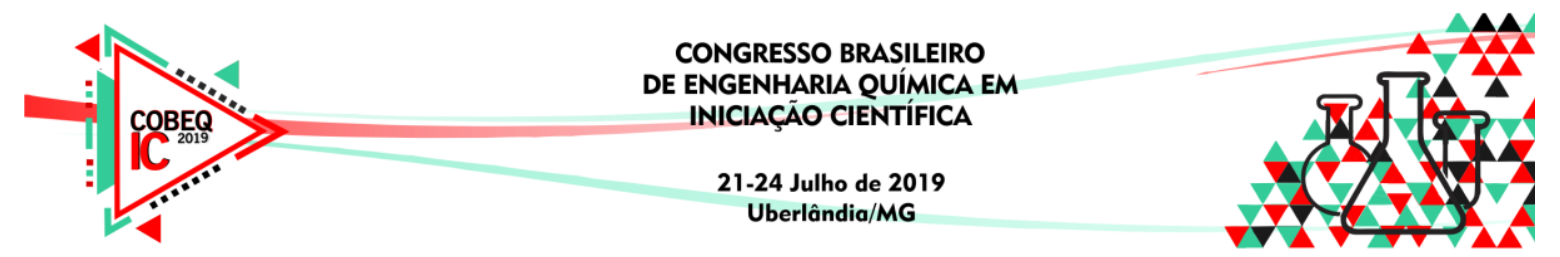

\section{METODOLOGIA}

\subsection{TRATAMENTO E CARACTERIZAÇÃO DE ADSORVENTE}

A matéria-prima foi previamente carbonizada, posteriormente lavada com água destilada para retirada de impurezas. A amostra foi seca a $100^{\circ} \mathrm{C}$ durante 24 horas. O material foi reduzido de tamanho em moinho de bolas e classificado em uma peneira de 60 mesh.

O preparo do carvão se baseou no melhor resultado observado por Li et al., (2011) com alterações. Nesta metodologia não foi realizada a hidrólise da matéria prima seguindo a seguinte rota: impregnação de ácido fosfórico $\left(\mathrm{H}_{3} \mathrm{PO}_{4}\right)$ concentrado $(85 \%)$ em uma proporção de $(1: 1)$, logo após o material calcinou em um forno mufla durante meia hora a $500^{\circ} \mathrm{C}$. Lavou-se com água deionizada o carvão afim de retirar o excesso do ácido e deixou para secar na estufa por $24 \mathrm{~h}$ a $105^{\circ} \mathrm{C}$. O material seco e calcinado foi caracterizado através de microscopia eletrônica. O Ponto de Carga Zero (PCZ) do carvão também foi determinado através da metodologia descrita por Regalbuto e Robles (2004) com algumas alterações principalmente nos valores de pH inicial. Foram empregados 10 pontos experimentais, constituídos pela mistura de $50 \mathrm{mg}$ de carvão ativado a $70 \mathrm{~mL}$ de soluções aquosas, preparadas sob diferentes valores de $\mathrm{pH}$ no intervalo de 1 a 11 . Em seguida, tal mistura foi posta em agitação por $24 \mathrm{~h}$, onde ao fim do processo o $\mathrm{pH}$ final das soluções foi verificado. Haja vista que o PCZ corresponde ao intervalo onde o pH final mantém-se constante independentemente do $\mathrm{pH}$ inicial (Guilarduci et al., 2006), um gráfico comparativo entre tais parâmetros foi plotado.

\subsection{PREPARO DO ADSORVATO}

Para o preparo da solução mãe pesou-se $200 \mathrm{mg}$ do corante Eosina Y, diluiu-se em água destilada e transferiu-se para um balão de fundo chato de $1 \mathrm{~L}$, aferido, para solução de 200 ppm, A solução foi analisada por um espectrofotômetro UV 1800 (Shimadzu), com comprimento de onda igual a $516 \mathrm{~nm}$. Para determinar a curva de calibração, utilizou-se as concentrações de 50, 100, 150, 200 e 250 ppm da solução de corante. O modelo linear obtido que fornece os valores para as concentrações em função das absorbâncias está descrito pela equação $1\left(R^{2}=0,9827\right)$.

$$
C_{f}=\frac{A b s .-1,1565}{0,0619}
$$

\subsection{ADSORÇÃO EM BANHO FINITO}

Pesou-se $0,3 \mathrm{~g}$ de carvão dispostas em erlenmeyers de $125 \mathrm{~mL}$, O cronômetro iniciou-se no momento da adição de $100 \mathrm{~mL}$ da solução do corante, sendo utilizadas soluções de 200 ppm. O sistema foi realizado em batelada sob agitação de $36 \mathrm{~Hz}$ no banho infinito metabólico com tempos de $30 \mathrm{~s}, 1,2,5$ e 10 minutos.

Após o término do mesmo a solução foi filtrada com disco de filtro a vácuo acoplado em uma seringa. O filtrado foi disposto em tubos plásticos etiquetados e posteriormente feita a análise do espectrofotômetro das cinco amostras em cubetas. A porcentagem removida de 


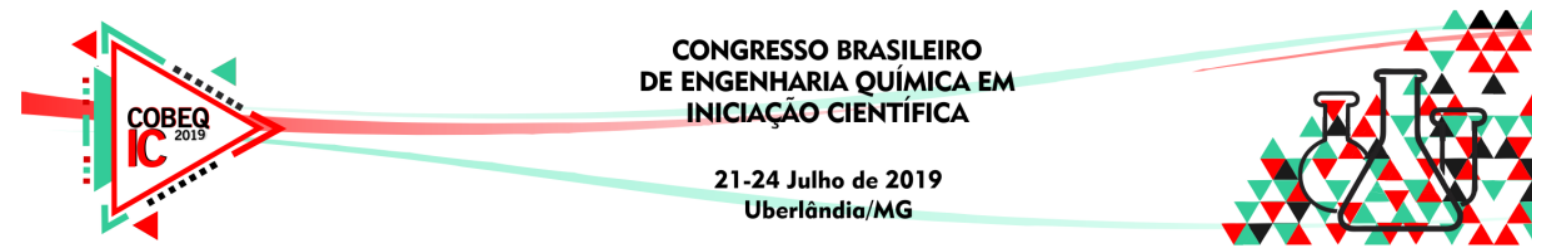

adsorvato e a quantidade adsorvida foram calculadas através das equações 2 e 3, respectivamente

$$
\begin{aligned}
& R=\frac{\left(C_{i}-C_{f}\right)}{C_{i}} \times 100 \\
& q_{t}=\frac{V\left(C_{i}-C_{f}\right)}{m}
\end{aligned}
$$

Cinética de adsorção: Após o fim dos testes de adsorção, determinou-se qual modelo cinético melhor se ajusta aos mesmos, sendo avaliados os modelos de pseudoprimeira ordem e pseudossegunda ordem, descritos pelas equações 4 e 5, respectivamente. A determinação dos parâmetros de cada modelo foi realizada através do software Origin, via regressão não linear.

$$
\begin{aligned}
& q_{t}=q_{e}\left[1-\exp \left(-k_{1} t\right)\right] \\
& q_{t}=\frac{q_{e}^{2} k_{2} t}{k_{2} q_{e} t+1}
\end{aligned}
$$

\section{RESULTADOS E DISCUSSÕES}

\subsection{CARACTERIZAÇÃO DO ADSORVENTE}

Microscopia Eletrônica de Varredura (MEV): A análise por microscopia eletrônica da casca de arroz in natura demonstra que há uma estrutura irregular constituída por poros, depressões e ranhuras, porém há presença de uma camada superficial sobrepondo os poros, dificultando a interação do adsorvato com os sítios ativos do adsorvente, reduzindo assim a eficiência do processo, como é ilustrado na figura 1a. Após o tratamento com agente químico e tratamento térmico, a figura $1 \mathrm{~b}$ revela que houve uma enorme redução da camada superficial, promovendo um aumento no número de poros e resultando em uma superfície heterogênea, propiciando uma melhor remoção do agente poluente.

Figura 1- Análise via MEV da casca de arroz in natura (a) e calcinada (b), com agente químico 


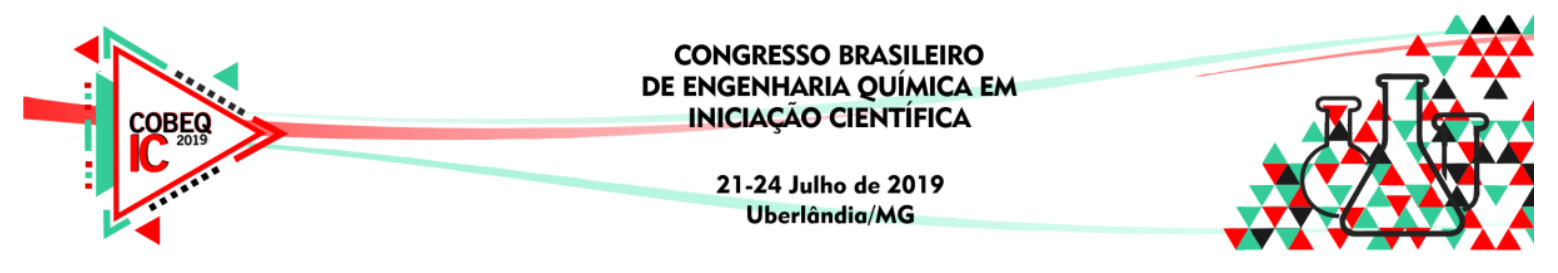

a)

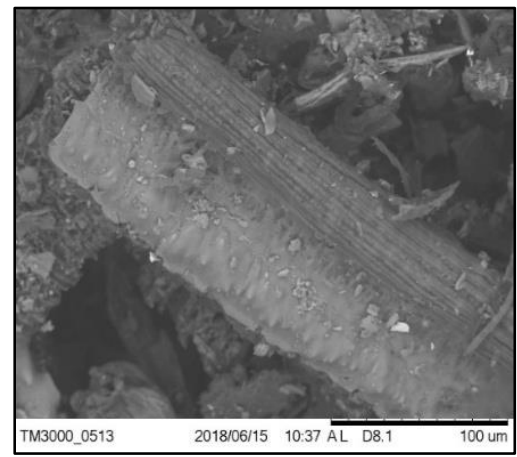

b)

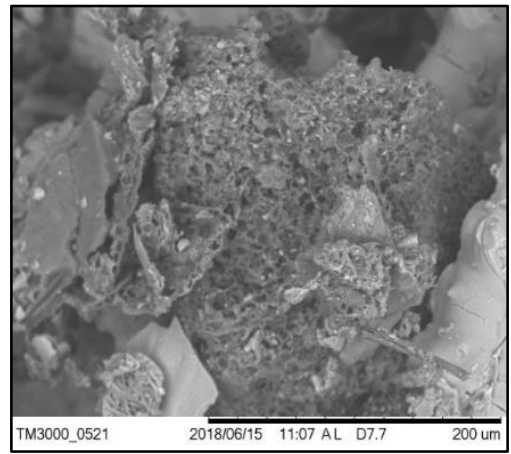

Determinação do Ponto de Carga Zero (PCZ): Os resultados obtidos para o teste do ponto de carga zero estão ilustrados na figura 1 , onde identifica-se que $\mathrm{pH} P C Z=3,28$. Nesse sentido, Ribeiro et al (2017) esclarece que ao haver o contato do adsorvente com uma solução aquosa com $\mathrm{pH}$ abaixo desse valor, a superfície será carregada positivamente e uma grande quantidade de ânions será adsorvida, enquanto que o contato do carvão com uma solução líquida cujo pH está acima de 3,28 irá carregar negativamente a superfície do sólido e haverá a adsorção de cátions. Todavia, Mimura et al (2010) produziu o adsorvente em questão, tratado com $\mathrm{NaOH}$, e obteve um valor para o $\mathrm{PCZ}$ próximo a 7. Portanto constata-se que a impregnação realizada por via $\mathrm{H}_{3} \mathrm{PO}_{4}$ influenciou no valor do $\mathrm{PCZ}$ obtido no presente trabalho.

Figura 3- Teste do ponto de carga zero para carvão ativado

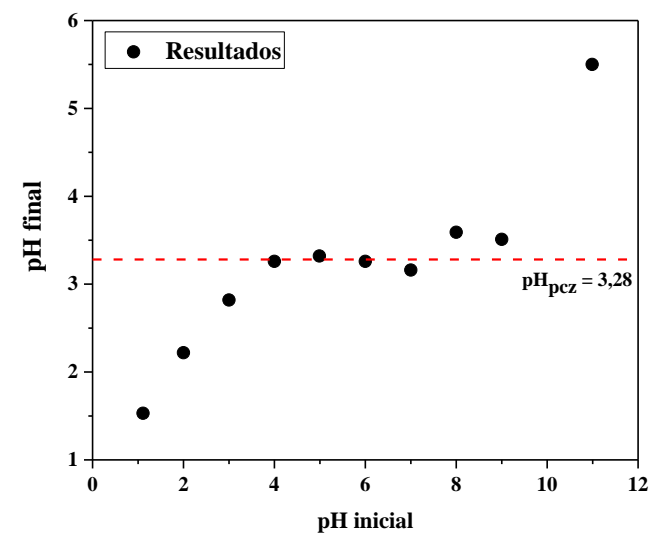

\subsection{CINÉTICA DE ADSORÇÃO}

Os resultados referentes à porcentagem removida de corante e a quantidade adsorvida do mesmo ao decorrer do tempo estão ilustrados na figura 4. Através das figuras $4 \mathrm{a}$ e $4 \mathrm{~b}$, verifica-se que os melhores resultados foram obtidos ao trabalhar-se a $\mathrm{pH}=4$, haja vista que neste cenário o carvão foi capaz de adsorver uma maior quantidade corante do meio, tendo sua estabilização antes de 10 min. Tal estabilização não é observada para o ajustado a pH $=8$, sendo necessário para esta condição um tempo maior de teste cinético. 


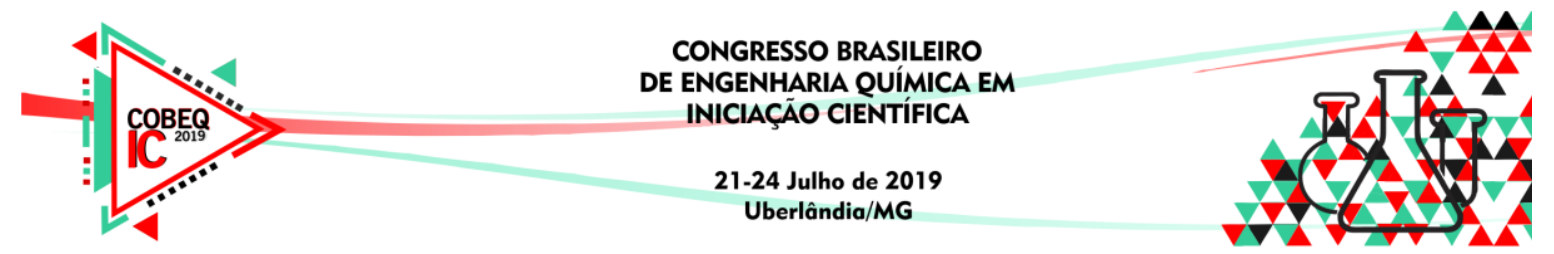

Figura 4 - Porcentagem removida (a) e quantidade adsorvida (b) de corante ao decorrer do tempo, sob diferentes $\mathrm{pHs}$

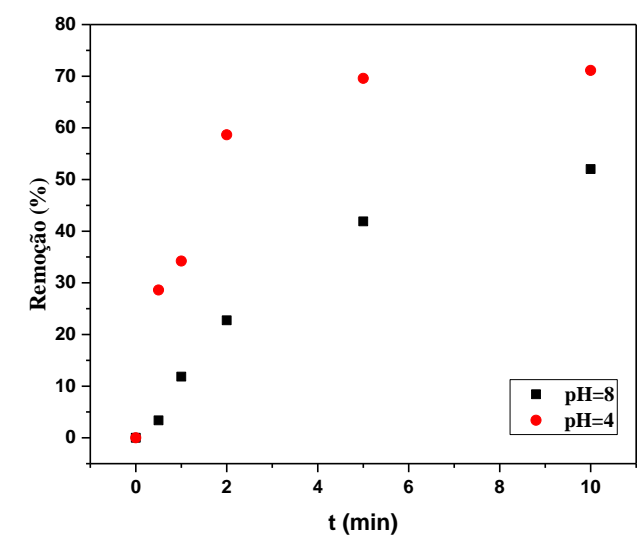

a)

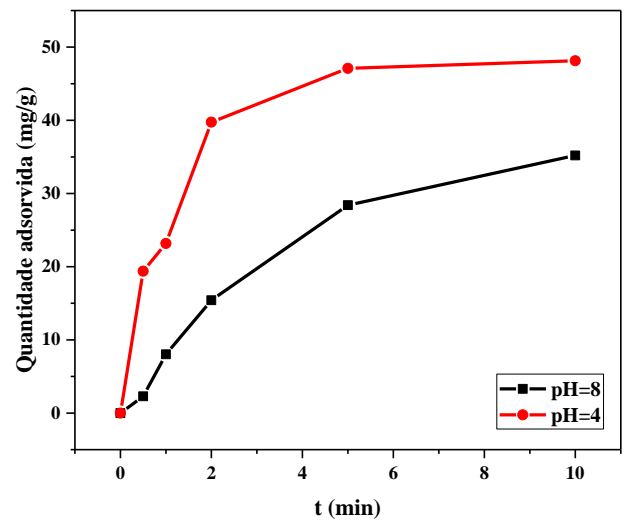

b)

Os parâmetros obtidos através para os modelos cinéticos estão dispostos na tabela 1 . Uma avaliação quantitativa dos modelos cinéticos, com base na comparação dos valores de $\mathrm{R}^{2}$ obtidos para cada regressão, revela que o modelo de pseudoprimeira ordem é o que melhor descreve o processo de adsorção ocorrido; tal modelo considera que a taxa de ocupação dos sítios de sorção será proporcional ao número de sítios desocupados (Xing et al, 2016). As curvas cinéticas ajustadas aos dados obtidos ao longo do experimento podem ser verificadas na figura 5 .

Tabela 1 - Parâmetros obtidos via regressão não linear para os modelos cinéticos analisados

\begin{tabular}{|c|c|c|c|c|c|c|}
\hline \multirow{2}{*}{$\mathrm{pH}$} & \multicolumn{3}{|c|}{ Pseudoprimeira ordem } & \multicolumn{3}{c|}{ Pseudossegunda ordem } \\
\cline { 2 - 7 } & $\mathrm{k}_{1}\left(\mathrm{~min}^{-1}\right)$ & $\mathrm{q}_{\mathrm{e}}(\mathrm{mg} / \mathrm{g})$ & $\mathrm{R}^{2}$ & $\mathrm{k}_{2}\left(\mathrm{~min}^{-1}\right)$ & $\mathrm{q}_{\mathrm{e}}(\mathrm{mg} / \mathrm{g})$ & $\mathrm{R}^{2}$ \\
\hline \hline 4 & 0,80898 & 71,04776 & 0,98586 & 0,01211 & 81,2728 & 0,98079 \\
\hline 8 & 0,24443 & 39,01499 & 0,994 & 0,00336 & 55,43303 & 0,9901 \\
\hline
\end{tabular}

Figura 5 - Curvas cinéticas a $\mathrm{pH}=4$ (a) e $\mathrm{pH}=8$ (b)

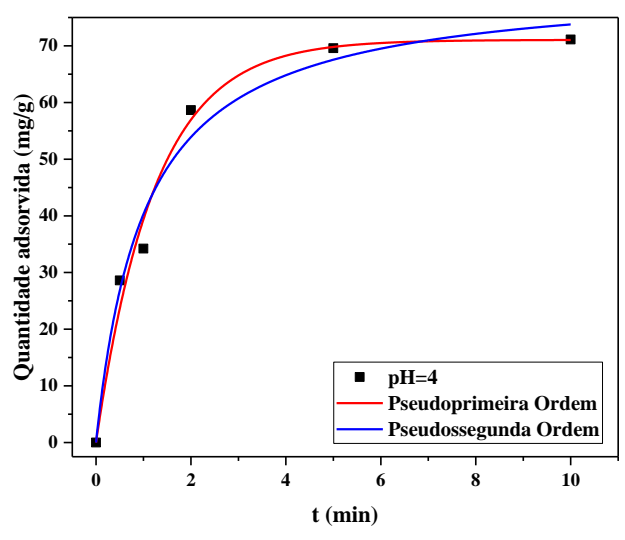

a)

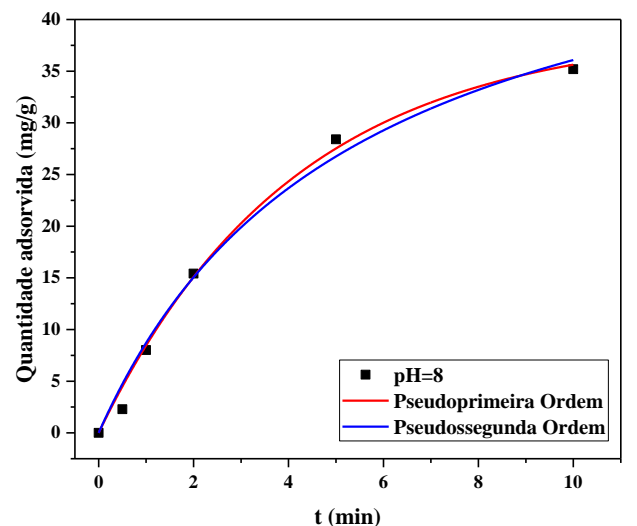

b) 


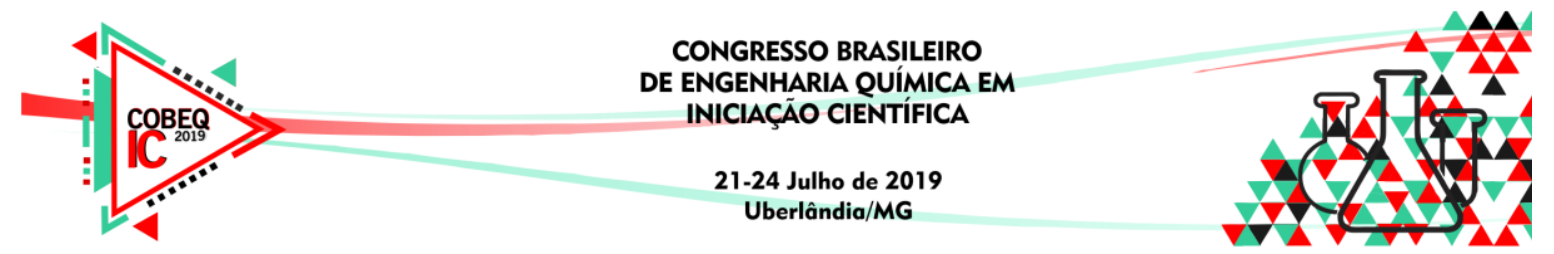

\section{CONCLUSÃO}

Logo, pode-se concluir ao fim deste trabalho que o carvão ativado da casca de arroz é um removedor eficiente do corante Eosina, em soluções aquosas.O modelo cinético de pseudoprimeira ordem se mostrou o melhor ajustado para os dois cenários avaliados; fatores como a concentração da solução e a faixa de $\mathrm{pH}$ analisada ser acima do PCZ podem justificar tal ocorrido. Ademais, nota-se que os melhores resultados foram obtidos em meios ácidos.

\section{REFERÊNCIAS BIBLIOGRÁFICAS}

ANITHA, T.; KUMAR, P. S.; KUMAR, K. S. Synthesis of nano-sized chitosan blended polyvinyl alcohol for the removal of Eosin Yellow dye from aqueous solution. Journal of Water Process Engineering, v. 13, p. 127-136, out. 2016.

GUILARDUCI, V. V. DA S. et al. Adsorção de fenol sobre carvão ativado em meio alcalino. Química Nova, v. 29, n. 6, p. 1226-1232, dez. 2006.

LI, et al. A new method of comprehensive utilization of rice husk. Journal hazardous materials 186 (2011) 2151-2156.

MIMURA, A. M. S. et al. Aplicação da casca de arroz na adsorção dos íons $\mathrm{Cu} 2+, \mathrm{Al} 3+, \mathrm{Ni} 2+\mathrm{e}$ Zn2+. Quimica Nova, v. 33, n. 6, p. 1279-1284, 2010.

NASCIMENTO, R. F., et al. Adsorção: aspectos teóricos e aplicações ambientais. Fortaleza: Impressa Universitária, p. 256, 2014.

REGAlBUTO, J. R.; ROBLES, J. O. The Engineering of Pt / Carbon Catalyst Preparation. University of Illinois , Chicago, 2004.

REIS, A. S., et al. Produção de carvão ativado a partir de casca de arroz. Desafios - Rev. Interdisciplinar da Universidade Federal do Tocantins, v. 2, p.89-103, 2015.

RIBEIRO, G. A. C. et al. Removal of Remazol brilliant violet textile dye by adsorption using rice hulls. Polímeros, v. 27, n. 1, p. 16-26, mar. 2017.

RUTHVEN, D. M. Principles of Adsorption and Adsorption Process. New York: John Wiley \& Sons, 1984

SOUSA NETO, V. O. Modificação química da casca do coco bruto (Cocos Nucifera) para remoção de $\mathrm{Cu}$ (II) de efluente sintético e industrial: estudo de isoterma de adsorção, cinética e coluna de leito fixo. 2012. 161 f. Tese (Doutorado em Engenharia Hidráulica e Ambiental) -Centro de Tecnologia, Universidade Federal do Ceará, Fortaleza, 2012.

XING, B. et al. Engineered Nanoparticles and the Environment: Biophysicochemical Processes and Toxicity. Hoboken, NJ, USA: John Wiley \& Sons, Inc., 2016. 